\title{
Symmetric Positive-Definite Constitutive Matrices for Discrete Eddy-Current Problems
}

\author{
Lorenzo Codecasa $^{1}$, Ruben Specogna ${ }^{2}$, and Francesco Trevisan ${ }^{2}$ \\ ${ }^{1}$ Dipartimento di Elettronica e Informazione, Politecnico di Milano, 20133 Milan, Italy \\ ${ }^{2}$ Dipartimento di Ingegneria Elettrica, Gestionale e Meccanica, Università di Udine, 33100 Udine, Italy
}

\begin{abstract}
We examine the construction of a symmetric positive definite conductance matrix for eddy-current problems, using a discrete approach. We construct a new set of piecewise uniform basis vector functions on both the primal and the dual complex. We define these vector functions for both tetrahedra and prisms.
\end{abstract}

Index Terms—Cell method, constitutive matrices, discrete approaches, eddy currents.

\section{INTRODUCTION}

$\mathbf{I}$ $\mathrm{N}$ discrete approaches [1]-[4] for eddy-current problems, the conductance matrix can be constructed geometrically according to different techniques proposed in [5] or in [9], but it is nonsymmetric. This fact leads to nonsymmetric stiffness matrices when solving the eddy-current problems. Moreover, these techniques hold only for the case of tetrahedra as primal volumes.

The motivation of this paper is to show a general method, extending [6] and [7], to construct consistent, symmetric positive-definite constitutive matrices for eddy-current problems, based on a new set of piecewise uniform basis vector functions defined both on the primal complex and on the dual complex. These vector basis functions will be introduced for tetrahedra and prisms with triangular base. In particular, we will introduce vector basis functions on the dual complex and for prisms where Whitney vector functions are not defined.

A numerical example will be used to compare the results obtained using different conductance matrices, constructed on both the primal and the dual cell complex based on tetrahedra as primal volumes.

\section{DISCRETE APPROACH FOR EDDY CURRENTS}

In this section, we will briefly recall the basic ideas of a discrete approach to solve eddy-current problems. The domain of interest $D$ of the eddy-current problem can be partitioned into a source region $D_{s}$, consisting of a current driven coil, a passive conductive region $D_{c}$, and an insulating region $D_{a}$ which is the complement of $D_{c}$ and $D_{s}$ in $D$. We introduce in $D$ a pair of interlocked cell complexes [8], [1], [2]. The primal complex consists of inner oriented cells such as nodes $n$, edges $e$, faces $f$, and volumes $v$. We will consider as primal volumes $v$ both tetrahedra and prisms with triangular base. ${ }^{1}$

\section{Digital Object Identifier 10.1109/TMAG.2006.887065}

Color versions of Figs. 1-5 are available online at http://ieexplore.iee.org.

${ }^{1}$ The lateral edges of the prism are not necessarily orthogonal to the base of the prism.
The dual complex is obtained from the primal according to the barycentric subdivision, with outer oriented cells such as dual volumes ${ }^{2} \tilde{n}$, dual faces $\tilde{e}$, dual edges $\tilde{f}$, and dual nodes $\tilde{v}$. For example, a dual node $\tilde{v}$ is the barycenter of the volume $v$.

The interconnections between cells of the primal complex, are defined by the usual connectivity matrices $\mathbf{G}$ between pairs $(e, n), \mathbf{C}$ between pairs $(f, e)$, and $\mathbf{D}$ between pairs $(v, f)$. Similarly, the corresponding matrices for the dual complex are $-\mathbf{G}^{T}$ (the minus sign is due to the assumption that a dual volume $\tilde{n}$ is oriented by the outward normal, while a node $n$ is oriented as a sink) between pairs $(\tilde{n}, \tilde{e}), \mathbf{C}^{T}$ between pairs $(\tilde{e}, \tilde{f})$, and $\mathbf{D}^{T}$ between pairs $(\tilde{f}, \tilde{v})$. With respect to these cell complexes, we recall the $A-\chi$ formulation [9], [10]. We search for the array A of the circulations $A$ of the magnetic vector potential along primal edges $e$ of $D$ and for the array $\chi$ of scalar potential $\chi$ associated with primal nodes $n$ of $D_{c}$ such that

$$
\begin{aligned}
& \left(\mathbf{C}^{T} \boldsymbol{\nu} \mathbf{C ~ A}\right)_{e}=(\mathbf{I})_{e} \quad \forall e \in D-D_{c} \\
& \left(\mathbf{C}^{T} \boldsymbol{\nu} \mathbf{C ~ A}\right)_{e}+i \omega\left(\boldsymbol{\sigma} \mathbf{A}_{c}\right)_{e}+i \omega(\boldsymbol{\sigma} \mathbf{G} \boldsymbol{\chi})_{e}=0 \quad \forall e \in D_{c} \\
& i \omega\left(\mathbf{G}^{T} \boldsymbol{\sigma} \mathbf{A}_{c}\right)_{n}+i \omega\left(\mathbf{G}^{T} \boldsymbol{\sigma} \mathbf{G} \boldsymbol{\chi}\right)_{n}=0 \quad \forall n \in D_{c}
\end{aligned}
$$

where array $\mathbf{A}_{c}$ is the sub-array of $\mathbf{A}$, associated with primal edges in $D_{c}$; the matrix $\mathbf{G}$ is associated with pairs $(e, n)$ of $D_{c}$. With $(\mathbf{x})_{k}$ we mean the $k$ th row of array $\mathbf{x}$, where $k=\{e, n\}$ is the label of edge $e$ or of node $n$. The array of currents crossing the dual faces is denoted by $\mathbf{I}$; if $e \in D_{s}$ then $(\mathbf{I})_{e}$ is the source current crossing the dual face $\tilde{e}$, while if $e \in D_{a}$ then $(\mathbf{I})_{e}=0$. Finally, $\boldsymbol{\nu}\left(\operatorname{dim}(\boldsymbol{\nu})=N_{f}, N_{f}\right.$ being the number of faces in $\left.D\right)$ is the reluctance constitutive matrix and $\boldsymbol{\sigma}$ is the conductance matrix $\left(\operatorname{dim}(\boldsymbol{\sigma})=N_{e c}, N_{e c}\right.$ being the number of edges in $\left.D_{c}\right)$. The system (1) is singular and to solve it we rely on CG method without gauge condition [12]. In system (1) the last set of equations may be eliminated, $\chi$ being arbitrary, and the so-called $A$-formulation [14] may be obtained as a particular case; however, the convergence of $A$-formulation depends strongly on the choice of the preconditioner as shown in [15], [16]. In the paper, we used a SSOR preconditioner and solving the singular system (1) provides a reduction of the effective condition number [14].

\footnotetext{
${ }^{2}$ This notation underlines the duality between a $p$-cell and its dual $(3-p)$-cell.
} 
In the case of tetrahedra, the techniques described in [5] or [13] based on Whitney vector functions can be used to construct constitutive matrices. However, these techniques lead to nonsymmetric matrices and cannot be used in the case of prisms.

In this paper, we will reformulate in a general way the approach described in [7] in order to treat tetrahedra or prisms in the same way.

\section{APPROACH FOR BUILDING CONSTITUTIVE MATRICES}

In this section, we provide a recipe to construct constitutive matrices. To this aim, we will need different sets of vector basis functions that will be defined in the next section. Here, we will give the specifications they have to comply with. We will construct the constitutive matrix at element level and then the global matrix will be assembled by adding the contributions from the single elements. Let $v_{k}$ be one primal volume, either a tetrahedron or a prism and $r_{i}$ be one of the following geometric entities: $e_{i}, f_{i}, \tilde{e}_{i}, \tilde{f}_{i}$ of $v_{k}$; we observe that $\tilde{e}_{i}, \tilde{f}_{i}$ are pieces of the $i$ th dual face or dual edge respectively, tailored in $v_{k}$. A generic vector function attached to $r_{i}$ is denoted by $v_{i}^{r}$.

We denote by $\mathrm{x}$ a vector field and by $X$ its integral $X_{i}=$ $\int_{r_{i}} \mathrm{x} \cdot d \mathrm{r}$ on the geometric entity $r_{i}$; similarly $Y_{i}$ denotes the integral $Y_{i}=\int_{\tilde{r}_{i}} \mathrm{y} \cdot d \mathrm{r}$ of vector field $\mathrm{y}$ with respect to the geometric entity $\tilde{r}_{i} d u a l^{3}$ to $r_{i}$. These are the so-called degrees of freedom (DoF). For example, if $\mathrm{x}$ is the electric displacement $\mathrm{D}$, then $X_{i}$ is the flux of D crossing dual face $\tilde{e}_{i}$. Similarly if y is the electric field $\mathrm{E}$, then $Y_{i}$ indicates the electromotive force (EMF) along primal edge $e_{i}$.

Using vector basis function $\mathrm{v}_{i}^{r}$ associated with geometric entity $r_{i}$, we may express field $\mathrm{x}$ as

$$
\mathrm{x}=\sum_{i \in \mathcal{R}} \mathrm{v}_{i}^{r} X_{i}
$$

where the subscript $i$ spans the set of labels $\mathcal{R}$ of the geometric entities $r_{i}$ in $v_{k}$. For example, if $r_{i}$ is a generic primal edge, then $\mathcal{R}$ is the set of the labels of the six primal edges of $v_{k}$.

Moreover, the elements of the set $\left\{\mathrm{v}_{i}^{r}\right\}$ have to comply at least with the following specifications:

i) they form a basis, so that $\int_{r_{j}} \mathrm{v}_{i}^{r} \cdot d \mathrm{r}=\delta_{i j}$;

ii) they can represent a uniform field exactly.

Now, we consider in $v_{k}$ the following functional:

$$
F=\frac{1}{2} \int_{v_{k}} \mathrm{x}^{\prime} \cdot \mathrm{y} d v
$$

where $\mathrm{x}^{\prime}, \mathrm{y}$ are a pair of vector fields in $v_{k}$ whose integrals $X_{i}^{\prime}$ and $Y_{i}$ are associated respectively with the geometric entities $r_{i}, \tilde{r}_{i}$ one dual of the other; the prime is used to stress that $\mathrm{x}^{\prime}, \mathrm{y}$ are independent fields, not necessarily related by a constitutive relation. Only the pair of fields $\mathrm{x}, \mathrm{y}$ is related by a constitutive relation of the kind $\mathrm{y}=m \mathrm{x}$ (or its inverse), $m$ being the material property.

\footnotetext{
${ }^{3}$ For example, the dual to edge $e_{i}$ is the dual face $\tilde{e}_{i}$ and the dual to dual edge $\tilde{f}_{i}$ is the primal face $f_{i}$
}

We assume that the field $\mathrm{x}^{\prime}$ in (3) is given as in (2), $\mathrm{x}^{\prime}=$ $\sum_{i \in \mathcal{R}} \mathrm{v}_{i}^{r} X_{i}^{\prime}$, the set $\left\{X_{i}^{\prime}\right\}$ being arbitrary. Then (3) becomes

$$
F=\frac{1}{2} \sum_{i \in \mathcal{R}} X_{i}^{\prime} \int_{v_{k}} \mathrm{v}_{i}^{r} \cdot \mathrm{y} d v
$$

At this stage, we may design basis functions such that the following equality

$$
\int_{v_{k}} \mathrm{v}_{i}^{r} \cdot \mathrm{y} d v=Y_{i}
$$

holds, and in this case the functional (3) can be written as

$$
F=\frac{1}{2} \sum_{i} X_{i}^{\prime} Y_{i}
$$

where the $X_{i}^{\prime}, Y_{i}$ belong to independent sets of DoF.

We try to satisfy (5) exactly at least for an element-wise uniform field $\mathrm{y}$; it will hold only approximately for a general field. Then (5) yields

$$
\mathrm{y} \cdot \int_{v_{k}} \mathrm{v}_{i}^{r} d v=Y_{i}
$$

where the equality sign holds if the following property

$$
\text { iii) } \int_{v_{k}} \mathrm{v}_{i}^{r} d v=\tilde{\mathrm{r}}_{i}
$$

is satisfied; we denote by $\tilde{r}_{i}$ the vector associated with the geometric entity $\tilde{r}_{i}$. It should be noted that this property reformulates in a purely geometric way, for a uniform material property $m$, the consistency condition firstly presented in [17] and recalled in [18] and [19].

The next step is to write $\mathrm{y}=m \mathrm{x}=m \sum_{j \in \mathcal{R}} \mathrm{v}_{j}^{r} X_{j}$, where we used (2) for $\mathrm{x}$. Substituting it in (4) and comparing with (6) we obtain

$$
Y_{i}=\sum_{j \in \mathcal{R}} \int_{v_{k}} \mathrm{v}_{i}^{r} \cdot m v_{j}^{r} d v X_{j}
$$

the set of $X_{i}^{\prime}$ being arbitrary and independent of the set of $X_{j}$. We observe that (8) is exact when the field y is element-wise uniform and property iii) holds. The numbers $\mathbf{M}_{i j}=\int_{v,} \mathbf{v}_{i}^{r}$. $m v_{j}^{r} d v$ are the entries of a constitutive matrix $\mathbf{M}$ that is by construction symmetric and positive-definite, relating the DoF arrays $\mathbf{Y}$ and $\mathbf{X}$ in the primal volume $v_{k}$.

\section{VECTOR BASIS FUnCTIONS}

In this section, we will construct the vector basis functions $\mathrm{v}^{r}$ attached to the geometric entity $r_{i}$ of volume $v_{k}$. Then, the vector functions are respectively: $\mathrm{v}_{i}^{e}$, attached to primal edge $e_{i}$, $\mathrm{v}_{i}^{\tilde{e}}$, attached to dual face $\tilde{e}_{i}, \mathrm{v}_{i}^{f}$, attached to primal face $f_{i}$ and $\mathrm{v}_{i}^{f}$, attached to dual edge $\tilde{f}_{i}$.

We introduce a generic subregion, say $\tau_{a}$, of volume the $v_{k}$ (either a tetrahedron or a prism), resulting from the intersec- 


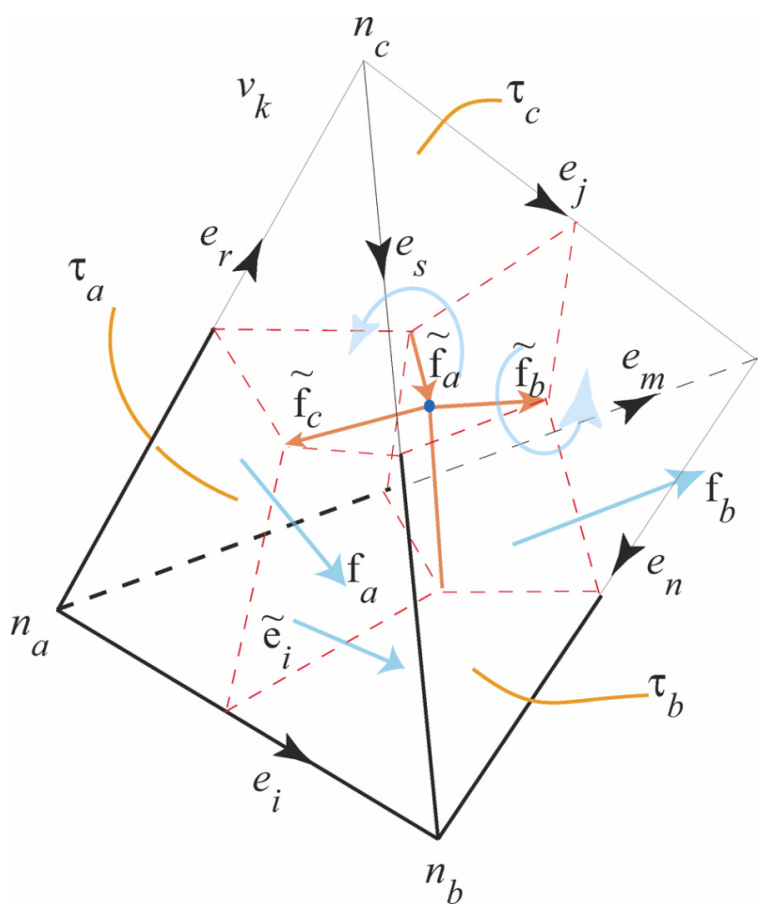

Fig. 1. Representation of the support of $\mathbf{v}_{i}^{e}$ and of $\tilde{\mathbf{v}}_{i}^{e}$. With the orientations shown, for edge vector function, we have $\mathrm{v}_{i}^{e}\left(\tau_{a}\right)=(-1)(-1) \mathrm{f}_{a} / 3 \mathrm{~V}$ and $\mathrm{v}_{i}^{e}\left(\tau_{b}\right)=(+1)(+1) \mathrm{f}_{b} / 3 \mathrm{~V}$. For dual face vector function, we have $\mathrm{v}_{i}^{\tilde{e}}\left(\tau_{a}\right)=$ $(-1)(-1)(12 / \mathrm{V}) \tilde{\mathbf{f}}_{a}$ and similarly $\mathbf{v}_{i}^{\tilde{e}}\left(\tau_{b}\right)=(+1)(+1)(12 / \mathrm{V}) \tilde{\mathbf{f}}_{b}$.

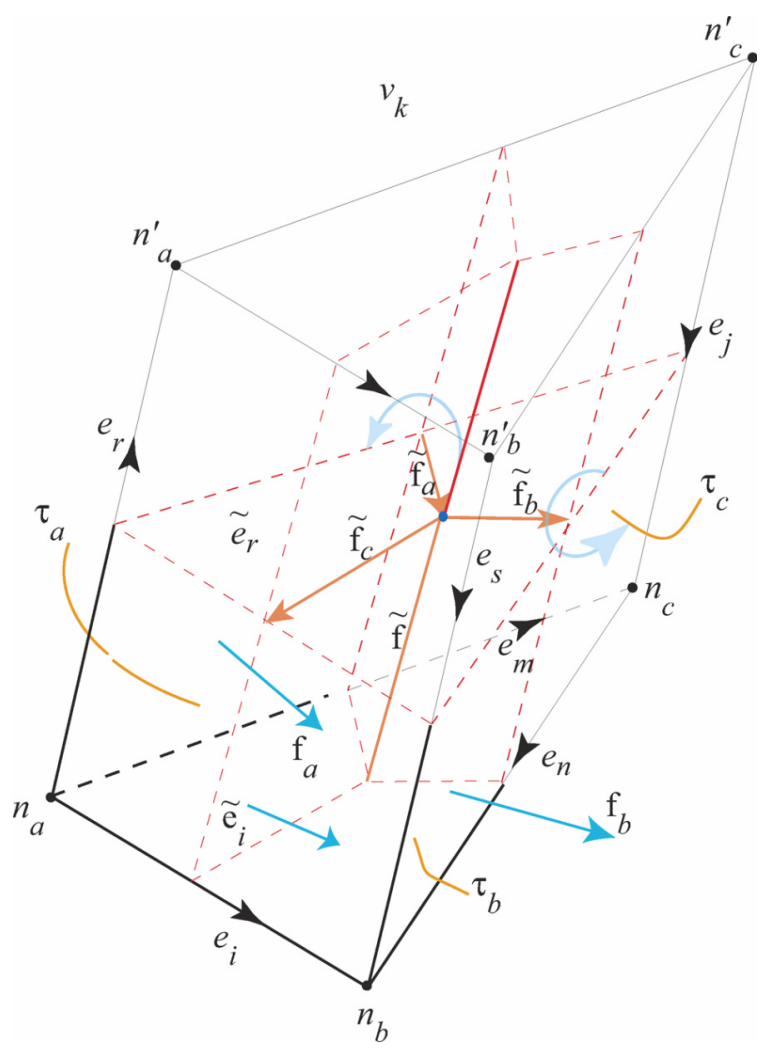

Fig. 2. Representation of the support of $\mathbf{v}_{i}^{e}$ and of $\tilde{\mathbf{v}}_{i}^{e}$. With the orientations shown, for edge vector function, we have $\mathrm{v}_{i}^{e}\left(\tau_{a}\right)=(-1)(-1) \mathrm{f}_{a} / 2 \mathrm{~V}$ and $\mathbf{v}_{i}^{e}\left(\tau_{b}\right)=(+1)(+1) \mathbf{f}_{b} / 2 \mathrm{~V}$. For dual face vector function, we have $\mathbf{v}_{i}^{\tilde{e}}\left(\tau_{a}\right)=$ $(-1)(-1)(12 / \mathrm{V}) \tilde{\mathbf{f}}_{a}$ and similarly $\mathrm{v}_{i}^{\tilde{e}}\left(\tau_{b}\right)=(+1)(+1)(12 / \mathrm{V}) \tilde{\mathbf{f}}_{b}$.

tion between $v_{k}$ and a dual volume $\tilde{n}_{a}$, see Figs. 1-3 for a detailed view; geometrically $\tau_{a}$ is always a hexahedron and it is

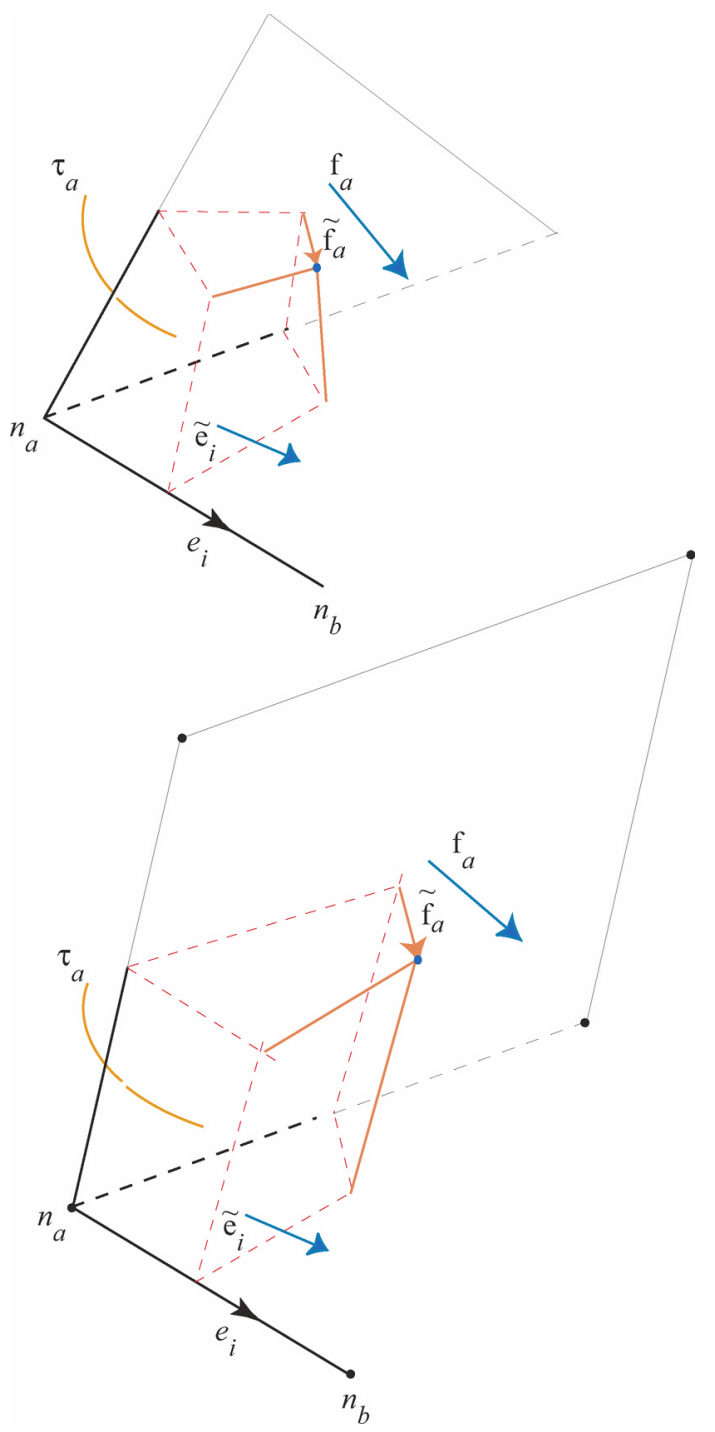

Fig. 3. Representation of the generic subregion $\tau_{a}$ one-to-one with node $n_{a}$ for a tetrahedron and a prism.

in a one-to-one correspondence with the primal node $n_{a}$. As a general rule, the support of $\mathrm{v}_{i}^{r}$ is $S_{r_{i}}=\bigcup_{s} \tau_{s}$, where $\tau_{s}$ belongs to the set of subregions having $r_{i}$ in common.

\section{A. Primal Edge Vector Functions}

The support of $\mathrm{v}_{i}^{e}$ is $S_{e_{i}}=\tau_{a} \bigcup \tau_{b}$ and $n_{a}, n_{b}$ are the boundary nodes of the primal edge $e_{i}$, Figs. 1, 2.

We denote by $f_{a}, f_{b}$ the faces containing $n_{a}$ and $n_{b}$ respectively and not containing $e_{i}$ and we introduce the area vectors $\mathrm{f}_{a}^{4}, \mathrm{f}_{b}$. Then $\mathrm{v}_{i}^{e}$ is defined as

$$
\mathrm{v}_{i}^{e}(p)= \begin{cases}\mathbf{G}_{i a} \mathbf{D}_{k a} \frac{1}{l_{1} \mathrm{~V}} \mathrm{f}_{a}, & \text { if } p \in \tau_{a} \\ \mathbf{G}_{i b} \mathbf{D}_{k b} \frac{1}{l_{1} \mathrm{~V}} \mathrm{f}_{b}, & \text { if } p \in \tau_{b}\end{cases}
$$

where $\mathbf{G}_{i a}$ is the incidence number between $e_{i}$ and $n_{a}, \mathbf{D}_{k a}$ is the incidence number between $v_{k}$ and $f_{a}, \mathrm{~V}$ is the volume of $v_{k}$; in the case of tetrahedra $l_{1}=3$, Fig. 1 . For prisms $l_{1}=2$

${ }^{4}$ Area vector $\mathrm{f}_{a}$ is normal to face $f_{a}$, its length is the area of $f_{a}$ and it points in a way congruent with the screw rule with respect to the inner orientation of the face. 
if $e_{i}$ belongs to a base, otherwise $l_{1}=1$ holds, Fig. 2 . In other words, $\mathrm{v}_{i}^{e}$ is a uniform vector in each subregion; for example in $\tau_{a}$ it is proportional to the area vector associated with the primal face opposite to edge $e_{i}$, see Fig. 3 .

It can be easily verified that Properties i) and ii) hold. Property iii) (see Figs. 1 and 2) follows from $\int_{v_{b}} \mathrm{v}_{i}^{e} d v=\left(\mathrm{f}_{a} / l_{1} \mathrm{~V}\right)\left(\mathrm{V} / l_{2}\right)+\left(\mathrm{f}_{b} / l_{1} \mathrm{~V}\right)\left(\mathrm{V} / l_{2}\right)$, where $l_{2}=4$ for tetrahedra and $l_{2}=6$ for prisms, and from the geometric identity ${ }^{5} \mathbf{G}_{i a} \mathbf{D}_{k a} \mathrm{f}_{a}+\mathbf{G}_{i b} \mathbf{D}_{k b} \mathrm{f}_{b}=l_{1} l_{2} \tilde{\mathrm{e}}_{i}$ where $\tilde{\mathrm{e}}_{i}$ is the area vector of the portion of the dual face $\tilde{e}_{i}$ tailored in $v_{k}$.

\section{B. Dual Face Vector Functions}

The support of $\mathrm{v}_{i}^{\tilde{e}}$ associated with a portion of dual face $\tilde{e}_{i}$ in $v_{k}$ is $S_{\tilde{e}_{i}}=\tau_{a} \bigcup \tau_{b}$, where subregions $\tau_{a}$ and $\tau_{b}$ have $\tilde{e}_{i}$ as common face, Figs. 1, 2. Then $\mathrm{v}_{i}^{\tilde{e}}$ is defined as

$$
\mathrm{v}_{i}^{\tilde{e}}(p)= \begin{cases}\mathbf{G}_{i a} \mathbf{D}_{k a} \frac{\tilde{l}_{1}}{\mathrm{~V}} \tilde{\mathrm{f}}_{a}, & \text { if } p \in \tau_{a} \\ \mathbf{G}_{i b} \mathbf{D}_{k b} \frac{\tilde{l}_{1}}{\mathrm{~V}} \tilde{\mathrm{f}}_{b}, & \text { if } p \in \tau_{b}\end{cases}
$$

where $\tilde{\mathrm{f}}_{a}, \tilde{\mathrm{f}}_{b}$ are the edge vectors associated respectively with the portions of dual edges $\tilde{f}_{a}, \tilde{f}_{b}$ in $v_{k}$. For any dual face of a tetrahedron and dual faces like $\tilde{e}_{i}$ in a prism (see Fig. 2) $\tilde{l}_{1}=12$ holds, while for a dual face like $\tilde{e}_{r}$ in a prism, we have $\tilde{l}_{1}=6$. In other words, $v_{i}^{\tilde{e}}$ is a uniform vector in each subregion; for example in $\tau_{a}$ it is proportional to the edge vector associated with the dual edge opposite to the portion of dual face $\tilde{e}_{i}$, see Fig. 3.

Obviously, property i), i.e., $\int_{\tilde{e}_{j}} \mathrm{v}_{i}^{\tilde{e}} \cdot d \mathrm{~s}=\delta_{i j}$ holds. For example, both for a tetrahedron and a prism $\int_{\tilde{e}_{i}} \mathrm{v}_{i}^{\tilde{e}_{i}} \cdot d \mathrm{~s}=1$, because $\tilde{\mathrm{f}}_{a} \cdot \tilde{\mathrm{e}}_{i}=\tilde{\mathrm{f}}_{b} \cdot \tilde{\mathrm{e}}_{i}=\mathrm{V} / 12$. In a prism, for dual faces like $\tilde{e}_{r}$ we have that $\tilde{\mathrm{f}}_{a} \cdot \tilde{\mathrm{e}}_{i}=\tilde{\mathrm{f}}_{b} \cdot \tilde{\mathrm{e}}_{i}=\mathrm{V} / 6$, where $\tilde{\mathrm{f}}_{a}, \tilde{\mathrm{f}}_{b}$ represent now the edge vectors associated with the portions of dual edges one-to-one with the top and bottom faces of the prism, respectively. Also property ii) holds.

Now, we will show that vector function $\mathrm{v}_{i}^{\tilde{e}}$ complies with property iii). We focus on $\mathrm{v}_{i}^{\tilde{e}}$ associated with the dual face like $\tilde{e}_{i}$ in a tetrahedron or in a prism, and we observe that $\int_{v} \mathrm{v}_{i}^{\tilde{e}} d v=$ $\left(\tilde{l}_{1} / V\right) \tilde{\mathrm{f}}_{a}\left(V / l_{2}\right)+\left(\tilde{l}_{1} / V\right) \tilde{\mathrm{f}}_{b}\left(V / l_{2}\right)$.

Let us consider, for simplicity, the tetrahedron with orientations shown in Fig. 1. Then, from elementary geometry, we have that $12 \tilde{\mathrm{f}}_{b}=\mathrm{e}_{i}+\mathrm{e}_{m}+\mathrm{e}_{r}, 12 \tilde{\mathrm{f}}_{a}=\mathrm{e}_{i}+\mathrm{e}_{n}+\mathrm{e}_{s}$ hold. Moreover, for faces $f_{a}$ and $f_{b}$, having edge $e_{j}$ in common, and the edge vectors of their bounding edges, we have $\mathrm{e}_{s}-\mathrm{e}_{n}=\mathrm{e}_{j}$ and $\mathrm{e}_{m}-$ $\mathrm{e}_{r}=\mathrm{e}_{j}$. Thus, we obtain $(12 / V) \tilde{\mathrm{f}}_{a}(V / 4)+(12 / V) \tilde{\mathrm{f}}_{b}(V / 4)=$ $1 / 2\left(\mathrm{e}_{i}+\mathrm{e}_{s}+\mathrm{e}_{r}\right)=\mathrm{e}_{i}$. A similar result holds for dual face vector functions associated with dual faces like $\tilde{e}_{i}$ or $\tilde{e}_{r}$ in a prism, see Fig. 2.

\section{Primal Face Vector Functions}

The support of $\mathrm{v}_{i}^{f}$ is the domain $S_{f_{i}}=\bigcup_{s} \tau_{s}$, where the boundary of volume $\tau_{s}$ and face $f_{i}$ have a non-null intersection. For example, in a tetrahedron and in a prism the support of $\mathrm{v}_{i}^{f}$ for a face having nodes $\left(n_{a}, n_{b}, n_{c}\right)$ (see Figs. 1 and 2) is $\tau_{a} \bigcup \tau_{b} \bigcup \tau_{c}$; on the other hand, for a face of a prism like $\left(n_{a}\right.$, $\left.n_{b}, n_{a}^{\prime}, n_{b}^{\prime}\right)$, the support is $\tau_{a} \bigcup \tau_{b} \bigcup \tau_{a^{\prime}} \bigcup \tau_{b^{\prime}}$.

${ }^{5}$ This identity holds when the dual complex is obtained with the barycentric subdivision.
Next, we denote by $\tau_{s}$ a generic subregion in the support $S_{f_{i}}$. We also denote by $\mathrm{e}_{s}$ the primal edge vector associated with edge $e_{s}$ drawn from the node $n_{s}$ and not belonging to the boundary of $f_{i}$. For example, considering the face having nodes $\left(n_{a}, n_{b}, n_{c}\right)$ in the tetrahedron or in the prism of Figs. 1 and 2, the edge is $e_{m}$, drawn from node $n_{a}$.

Now, we define $\mathrm{v}_{i}^{f}$ attached to $f_{i}$, as

$$
\mathrm{v}_{i}^{f}(p)=\mathbf{D}_{k i} \mathbf{G}_{r s} \frac{1}{l \mathrm{~V}} \mathrm{e}_{s}, \quad \text { if } p \in \tau_{s} \subset S_{f_{i}}
$$

where $l=3$ for a tetrahedron; in a prism, for a face like the one having nodes $\left(n_{a}, n_{b}, n_{c}\right), l=2$ while for a face like the one having nodes $\left(n_{a}, n_{b}, n_{a}^{\prime}, n_{b}^{\prime}\right), l=1$. The incidence numbers $\mathbf{D}_{k i}, \mathbf{G}_{r s}$, specify the incidence between the pairs $\left(v_{k}, f_{i}\right)$, $\left(e_{r}, n_{s}\right)$, respectively.

With a reasoning similar to the one presented in the previous sections, it is easy to show that properties i), ii), and iii) hold for $\mathrm{v}_{i}^{f}$.

\section{Dual Edge Vector Functions}

The support of a dual edge vector function $\mathrm{v}_{i}^{\tilde{f}}$ is $S_{\tilde{f}_{i}}=\bigcup_{s} \tau_{s}$, where $\tau_{s}$ has $\tilde{f}_{i}$ as common edge. For example, in a tetrahedron the support for a dual edge like $\tilde{f}_{c}$ (see Fig. 1) is $\tau_{a} \bigcup \tau_{b} \bigcup \tau_{c}$; on the other hand (see Fig. 2), for a dual edge of a prism like $\tilde{f}_{a}$, the support is $\tau_{a} \bigcup \tau_{c} \bigcup \tau_{a^{\prime}} \bigcup \tau_{c^{\prime}}$.

Next, we consider a generic subregion $\tau_{s}$ in the support $S_{\tilde{f}_{i}}$. We denote by $\tilde{e}_{s}$ the dual face having only the barycenter $\tilde{v}_{k}$ of $v_{k}$ in common with the dual edge $\tilde{f}_{i} ; \tilde{\mathrm{e}}_{s}$ is the corresponding dual face vector associated with $\tilde{e}_{s}$. For example, considering the dual edge $\tilde{f}_{c}$ and subregion $\tau_{a}$ in the tetrahedron or in the prism of Figs. 1 and 2 , the dual face is $\tilde{e}_{m}$.

Now, we define $\mathrm{v}_{i}^{\tilde{f}}$ in $\tau_{s}$, as

$$
\mathrm{v}_{i}^{\tilde{f}}(p)=\mathbf{D}_{k i} \mathbf{G}_{r s} \frac{\tilde{l}}{\mathrm{~V}} \tilde{\mathrm{e}}_{s}, \quad \text { if } p \in \tau_{s} \subset S_{\tilde{f}_{i}}
$$

where $\tilde{l}=12$ for a tetrahedron and a prism, for a dual edge like $\tilde{f}_{c}$; in a prism for a dual edge like $\tilde{f}, \tilde{l}=6$, and the incidence numbers $\mathbf{D}_{k i}, \mathbf{G}_{r s}$, refer to the pairs $\left(v_{k}, f_{i}\right),\left(e_{r}, n_{s}\right)$, respectively.

Again, with a reasoning similar to the one presented in the previous sections, it is easy to show that properties i), ii), and iii) hold for $\mathrm{v}_{i}^{\tilde{f}}$.

\section{Constitutive Matrices}

We will write the reluctance and conductance constitutive matrices explicitly by assigning to the general expressions $X_{j}$, $Y_{i}, m, \mathcal{R}$ and $\mathrm{v}_{i}^{r}$ in (8) the variables of the specific case. We denote by $\mathcal{E}$ the number of edges of $v_{k}$ and with $\mathcal{F}$ the number of its faces; $\mathcal{E}=6, \mathcal{F}=4$ for a tetrahedron while $\mathcal{E}=9, \mathcal{F}=5$ for a prism.

\section{A. Magnetic Matrix Using $\mathrm{v}_{i}^{f}$}

The reluctance matrix $\boldsymbol{\nu}$ for tetrahedron $v_{k}$ relates the induction fluxes $\Phi_{j}=X_{j}$ associated with $f_{j}$ with the magnetomotive forces (m.m.f.s) $F_{i}=Y_{i}$ associated with $\tilde{f}_{j}, \operatorname{dim}(\boldsymbol{\nu})=\mathcal{F}$. 
Then, the entries are $\boldsymbol{\nu}_{i j}=\int_{v_{k}} \mathrm{v}_{i}^{f} \cdot \nu \mathrm{v}_{j}^{f} d v$, where $\nu=m$ is the reluctivity of $v_{k}$ and $\mathcal{R}$ is the set of labels of the $\mathcal{F}$ primal faces of $v_{k}$.

\section{B. Magnetic Matrix Using $\mathrm{v}_{i}^{\tilde{f}}$}

As a first step, we construct matrix $\boldsymbol{\mu}$ for tetrahedron $v_{k}$ relating $F_{j}=X_{j}$ with $\Phi_{i}=Y_{i}, \operatorname{dim}(\boldsymbol{\mu})=\mathcal{F}$. Its entries are $\boldsymbol{\mu}_{i j}=\int_{v_{k}} \mathrm{v}_{i}^{\tilde{f}} \cdot \mu \mathrm{v}_{j}^{\tilde{f}} d v$, where $\mu=m$ is the permeability of $v_{k}$ and $\mathcal{R}$ is the set of labels of the $\mathcal{F}$ primal faces of $v_{k}$. The second step is to invert it and we obtain $\tilde{\boldsymbol{\nu}}=\mu^{-1}$, where the reluctance matrix $\tilde{\boldsymbol{\nu}}$ for tetrahedron $v_{k}$, relates the induction fluxes $\Phi_{j}=X_{j}$ associated with $f_{j}$ with m.m.f.s $F_{i}=Y_{i}$ associated with $\tilde{f}_{j}$.

\section{Conductance Matrix Using $\mathrm{v}_{i}^{e}$}

Conductance matrix $\boldsymbol{\sigma}$ for tetrahedron $v_{k}$ relates the EMFs $U_{j}=X_{j}$ associated with $e_{j}$ with currents $I_{i}=Y_{i}$ associated with $\tilde{e}_{j}, \operatorname{dim}(\boldsymbol{\sigma})=\mathcal{E}$. Then, the entries are $\boldsymbol{\sigma}_{i j}=\int_{v_{k}} \mathrm{v}_{i}^{e}$. $\sigma \mathrm{v}_{j}^{e} d v$, where $\sigma=m$ is the conductivity of $v_{k}$ and $\mathcal{R}$ is the set of labels of the $\mathcal{E}$ primal edges of $v_{k}$.

\section{Conductance Matrix Using $\mathrm{v}_{i}^{\tilde{e}}$}

Similarly, we construct matrix $\boldsymbol{\rho}$ for tetrahedron $v_{k}$ relating $I_{j}=X_{j}$ with $U_{i}=Y_{i}, \operatorname{dim}(\boldsymbol{\rho})=\mathcal{E}$ whose entries are $\boldsymbol{\rho}_{i j}=$ $\int_{v_{k}} \mathrm{v}_{i}^{\tilde{e}} \cdot \rho \mathrm{v}_{j}^{\tilde{e}} d v$, where $\rho=m$ is the resistivity of $v_{k}$ and $\mathcal{R}$ is the set of labels of the $\mathcal{E}$ primal edges of $v_{k}$. Then we invert it, obtaining $\tilde{\sigma}=\rho^{-1}$, where the conductance matrix $\tilde{\sigma}$ for tetrahedron $v_{k}$, relates the EMFs $U_{j}=X_{j}$ associated with $e_{j}$ with the currents $I_{i}=Y_{i}$ associated with $f_{j}$.

\section{NUMERICAL RESULTS AND COMPARISONS}

As numerical test, we consider a geometry consisting of a circular coil placed above an aluminum plate. The domain of interest $D$ of the eddy-current problem (a cylinder of diameter of $60 \mathrm{~mm}$ and height $44.5 \mathrm{~mm}$ ), has been partitioned into a source region $D_{s}$ (a circular current driven coil of $18 \mathrm{~mm}$ of outer diameter, $12 \mathrm{~mm}$ of inner diameter, and $10 \mathrm{~mm}$ height) placed above a conducting region $D_{c}$ consisting of an aluminium plate $4 \mathrm{~mm}$ thick and with a radius of $30 \mathrm{~mm}$. The insulating region $D_{a}$ is the complement of $D_{c}$ and $D_{s}$ in $D$. In $D_{s}$, we force a sinusoidal current source $I_{s}=\sin (\omega t)$ with a frequency of $f=5 \mathrm{kHz}$.

We assemble the system (1) using the conductance constitutive matrices $\boldsymbol{\sigma}, \tilde{\boldsymbol{\sigma}}$ for tetrahedra, respectively. For comparison, we also used the symmetric and nonsymmetric conductance constitutive matrices computed according to the methods described in previous papers [11] and [9], respectively; these methods make use of the Whitney edge vector functions [5], [13] and, therefore, they are limited to the primal complex. We solve the final system with a QMR solver for complex symmetric and nonsymmetric matrices according to the case; in both the cases, we use a SSOR preconditioner.

To study convergence, we start from a couple of meshes named Mesh 1 (10510 tetrahedra) and Mesh 2 (40643 tetrahedra). Then, we use the uniform refinement technique to produce finer meshes. This technique for anisotropic meshes provides more regular results. Therefore, by uniform refinement of Mesh 1 we obtain Mesh 3 (84080 tetrahedra) and

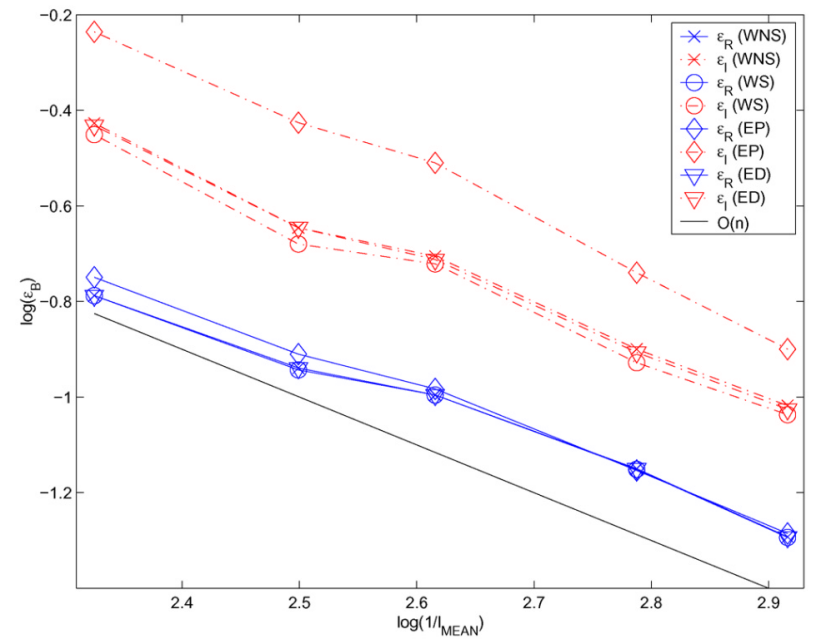

Fig. 4. Convergence rate of the error for the real (subscript R) and the imaginary (subscript I) parts of the magnetic induction calculated using various constitutive matrices (WNS $=$ Whitney non-symmetric, $\mathrm{WS}=$ Whitney symmetric, $\mathrm{EP}=\boldsymbol{\sigma}, \mathrm{ED}=\tilde{\boldsymbol{\sigma}}$ ). In both the axes we used a $\log$ scale, in abscissa the average length of the element is considered.

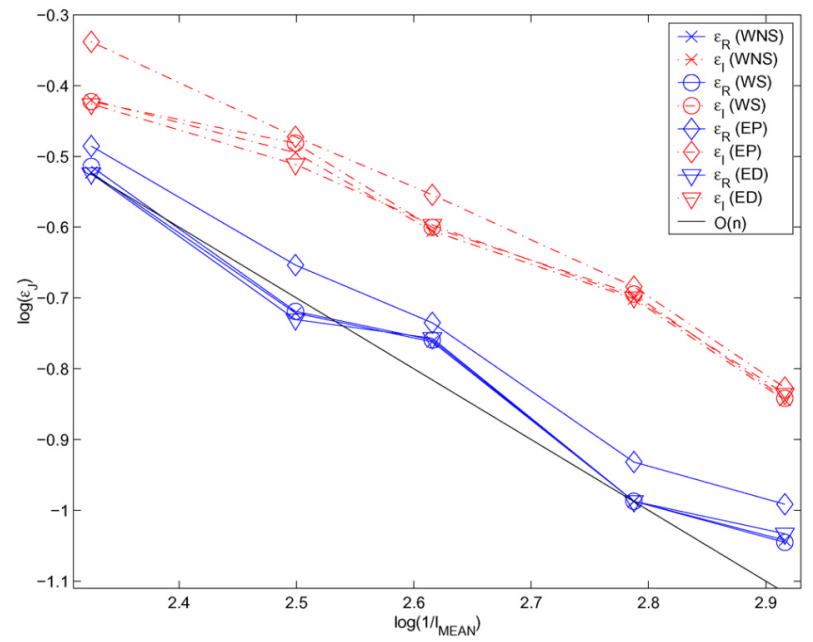

Fig. 5. Convergence rate of the error for the real (subscript $R$ ) and the imaginary (subscript I) parts of the eddy current density calculated using various constitutive matrices (WNS $=$ Whitney non-symmetric, $\mathrm{WS}=$ Whitney symmetric, $\mathrm{EP}=\boldsymbol{\sigma}, \mathrm{ED}=\tilde{\boldsymbol{\sigma}})$. In both the axes we used a $\log$ scale, in abscissa the average length of the element is considered.

Mesh 5 (672640 tetrahedra); similarly from the Mesh 2 (40643 tetrahedra) we obtain Mesh 4 (325144 tetrahedra).

Figs. 4 and 5 show the convergence rate of the magnetic induction and the current density with different constitutive matrices. We calculate the error in energy norm defined as

$$
\epsilon_{B}=\sqrt{\frac{\int_{D} \nu\left|B-B_{\mathrm{REF}}\right|^{2} d v}{\int_{D} \nu\left|B_{\mathrm{REF}}\right|^{2} d v}}
$$

where $B_{\mathrm{REF}}$ is the reference induction field computed by means of a 2-D axisymmetric finite-element accurate solution. As quality factor for the mesh, we choose the mean length of the edges. For comparison using Mesh 5, the CPU time (on a Pentium IV $2 \mathrm{GHz}$ ) needed to solve iteratively the linear system with a stop criterion on the residual 2-norm less then $10^{-6}$, 
is of $69 \mathrm{~min}$ and $9 \mathrm{~min}$, respectively, for nonsymmetric and symmetric conductance matrices obtained from Whitney edge vector functions, $7 \mathrm{~min}$ and $9 \min$ for $\boldsymbol{\sigma}$ and $\tilde{\boldsymbol{\sigma}}$, respectively.

\section{CONCLUSION}

We proposed an approach for both tetrahedra and prisms with triangular base which allows to construct symmetric positivedefinite constitutive matrices. The approach relies on a set of piecewise uniform vector basis functions defined in a fully geometric way on both the primal and the dual complex, where Whitney vector functions do not exist. This peculiarity makes the implementation straightforward and efficient. A numerical example evidences the convergence rate of the different approximated solutions and the significant saving of time when the proposed symmetric, positive-definite constitutive matrices are used instead of the the nonsymmetric matrices.

\section{REFERENCES}

[1] A. Bossavit, "How weak is the "weak solution" in finite element methods?," IEEE Trans. Magn., vol. 34, no. 5, pp. 2429-2432, Sep. 1998.

[2] A. Bossavit and L. Kettunen, "Yee-like schemes on staggered cellular grids: A synthesis between FIT and FEM approaches," IEEE Trans. Magn., vol. 36, no. 4, pp. 861-867, Jul. 2000.

[3] E. Tonti, "Algebraic topology and computational electromagnetism," in 4th Int. Workshop on Electric and Magnetic Fields, Marseille, France, May 12-15, 1988, pp. 284-294.

[4] M. Clemens and T. Weiland, "Transient eddy current calculation with the FI-method," IEEE Trans. Magn., vol. 35, no. 3, pp. 1163-1166, May 1999.

[5] T. Tarhasaari, L. Kettunen, and A. Bossavit, "Some realizations of a discrete Hodge operator: A reinterpretation of finite element techniques," IEEE Trans. Magn., vol. 35, no. 3, pp. 1494-1497, May 1999.
[6] L. Codecasa, V. Minerva, and M. Politi, "Use of barycentric dual grids for the solution of frequency domain problems by FIT," IEEE Trans. Magn., vol. 40, no. 2, pp. 1414-1419, Mar. 2004.

[7] L. Codecasa and F. Trevisan, "Piecewise uniform bases and energetic approach for discrete constitutive matrices in electromagnetic problems," Int. J. Numer. Methods Eng., vol. 65, no. 4, pp. 548-565, 2006.

[8] E. Tonti, "Finite formulation of electromagnetic field," IEEE Trans. Magn., vol. 38, no. 2, pp. 333-336, Mar. 2002.

[9] F. Trevisan, "3-D eddy current analysis with the cell method for NDE problems," IEEE Trans. Magn., vol. 40, no. 2, pp. 1314-1317, Mar. 2004.

[10] F. Trevisan and L. Kettunen, "Geometric interpretation of finite dimensional eddy current formulations," Int. J. Numer. Methods Eng., vol. 67, no. 13, pp. 1888-1908, 2006.

[11] R. Specogna and F. Trevisan, "Discrete constitutive equations in $A-\chi$ geometric eddy-current formulation," IEEE Trans. Magn., vol. 41, no. 4, pp. 1259-1263, Apr. 2005.

[12] A. Kameari and A. Koganezawa, "Convergence of ICCG method in FEM using edge elemements without gauge condition," IEEE Trans. Magn., vol. 33, no. 2, pp. 1223-1226, Mar. 1997.

[13] F. Trevisan and L. Kettunen, "Geometric interpretation of discrete approaches to solving magnetostatics," IEEE Trans. Magn., vol. 40, no. 2, pp. 361-365, Mar. 2004

[14] H. Igarashi and T. Honma, "On convergence of ICCG applied to finiteelement equation for quasi-static fields," IEEE Trans. Magn., vol. 38 , no. 2 , pp. 565-568, Mar. 2002.

[15] B. Weiss and O. Biro, "On the convergence of transient eddy-current problems,” IEEE Trans. Magn., vol. 40, no. 2, pp. 957-960, Mar. 2004.

[16] S. Reitzinger and M. Kaltenbacher, "Algebraic multigrid methods for magnetostatic field problems," IEEE Trans. Magn., vol. 38, no. 2, pp. 477-480, Mar. 2002.

[17] A. Bossavit, "Generating Whitney forms of polynomial degree one and higher," IEEE Trans. Magn., vol. 38, no. 2, pp. 341-344, Mar. 2002.

[18] M. Cinalli, F. Edelvik, R. Schuhmann, and T. Weiland, "Consistent material operators for tetrahedral grids based on geometrical principles," Int. J. Numer. Model., vol. 17, pp. 487-507, 2004.

[19] M. Marrone, "A new consistent way to build symmetric constitutive matrices on general 2-D grids," IEEE Trans. Magn., vol. 40, no. 2, pp. 1420-1423, Mar. 2004.

Manuscript received February 17, 2006; revised October 24, 2006. Corresponding author: F. Trevisan (e-mail: trevisan@uniud.it). 